\title{
The promising role of radiotherapy in the treatment of advanced or metastatic renal cell carcinoma: a narrative review
}

\author{
Qinghong Wei ${ }^{1 \#}$, Hongmei $\mathrm{He}^{2 \#}$, Long $\mathrm{Lv}^{3}$, Xiaoying $\mathrm{Xu}^{1}$, Weibing Sun ${ }^{3}$ \\ ${ }^{1}$ Department of Radiation Oncology, The Second Affiliated Hospital of Dalian Medical University, Dalian, China; ${ }^{2}$ Department of medical \\ Oncology, The Second Affiliated Hospital of Dalian Medical University, Dalian, China; ${ }^{3}$ Department of Urology, The Second Affiliated Hospital of \\ Dalian Medical University, Dalian, China \\ Contributions: (I) Conception and design: X Xu, W Sun; (II) Administrative support: H He; (III) Provision of study materials or patients: X Xu, W \\ Sun; (IV) Collection and assembly of data: Q Wei; (V) Data analysis and interpretation: Q Wei, L Lv; (VI) Manuscript writing: All authors; (VII) \\ Final approval of manuscript: All authors. \\ \#These authors contributed equally to this work as co-first authors. \\ Correspondence to: Xiaoying Xu; Weibing Sun. The Second Affiliated Hospital of Dalian Medical University, Dalian, China. \\ Email: xiaoyingxu73@aliyun.com; weibingsun_dyfemw@163.com.
}

\begin{abstract}
Traditionally, renal cell carcinoma (RCC) has been regarded to be "radioresistant". Conventional fractionated radiation (CFRT) has played a limited role in RCC as a palliative treatment to relieve pain and bleeding. Succeed to the rapid development of precise radiotherapy techniques, realizing safe delivery of high-dose radiotherapy, an increasing amount of convincing data suggests that the delivery of high-dose-perfraction radiation through stereotactic radiosurgery (SRS) or stereotactic body radiation therapy (SBRT), also known as stereotactic ablative radiotherapy (SABR) can help to overcome resistance to radiotherapy. Herein, we summarized and analyzed the data from randomized controlled trials, retrospective and prospective studies, and meta-analyses relating to the treatment of advanced and metastatic RCC (mRCC) with CFRT, SBRT, or SBRT combined with systemic therapy. CFRT has a limited effect on local control (LC) of advanced RCC and mRCC, but it is a major palliative treatment which could obviously relieve pain caused by cancer. SBRT and SRS have the significant advantage of being able to precisely deliver a high dose of radiation to the target tissues. SBRT could cause a higher LC for advanced and metastatic RCC and could be used as an alternative to surgery for patients with oligometastatic RCC. The combination of SBRT with systemic therapy, such as targeted therapy or immunotherapy, is safe and tolerable. Concurrent immunotherapy and SBRT is a promising treatment strategy for patients with advanced or metastatic RCC. However, research on radiotherapy combined with systemic therapy is still limited and further studies to explore this treatment for RCC are urgently needed.
\end{abstract}

Keywords: Renal cell carcinoma (RCC); conventional fractionated radiation (CFRT); stereotactic radiosurgery (SRS); stereotactic body radiation therapy (SBRT); targeted therapy; immunotherapy

Submitted Oct 23, 2020. Accepted for publication Dec 16, 2020.

doi: $10.21037 /$ tau-20-1466

View this article at: http://dx.doi.org/10.21037/tau-20-1466

\section{Introduction}

Renal cell carcinoma (RCC), which is the eighth most common malignancy among adults in the United States, is rising in incidence (1). In most cases of RCC, surgery is still an indispensable treatment option, and radical surgery or surgery combined with multiple therapies is still a standard strategy for treating patients with this malignancy (2). However, about $5 \%$ of RCC patients suffer from local recurrence and as many as $20-30 \%$ of patients progress to metastatic disease after undergoing surgery for localized lesions $(3,4)$. The response rate among patients 
with advanced disease is extremely low, and their survival rate is considerably reduced compared to that of patients with early-stage disease (5). National Comprehensive Cancer Network (NCCN) guidelines recommend the use of radiation as a palliative therapy, especially to relieve the pain caused by bone metastases. For patients with multifocal brain metastases (BM), whole-brain irradiation is recommended as an effective measure, whereas stereotactic body radiation therapy (SBRT) is a potential option for patients who undergo nephrectomy and experience oligometastatic recurrence years later (6).

Stereotactic radiosurgery (SRS) or fractionated stereotactic radiotherapy (FSRT) is a treatment modality through which a high dose of radiation can be delivered to target areas in single or multiple fractions, while minimizing the damage to normal tissue caused by radiation exposure (7). Ning et al. reported that high dose radiation could efficiently eradicate RCC cells in vitro (8). Several clinical studies on the use of SRS or SBRT to treat patients with primary or metastatic RCC (mRCC) have reported promising results. Furthermore, emerging preclinical and clinical data have suggested that local RT could supplement the effects of immunotherapy to enhance anti-tumor immunity (9). This review aimed to summarize the current evidence of the role of RT in the treatment of advanced or metastatic RCC from studies focusing on the use of CFRT, SRS, SBRT, or SBRT in combination with systemic therapy.

We present the following article in accordance with the Narrative Review reporting checklist (available at http:// dx.doi.org/10.21037/tau-20-1466).

\section{Methods}

Database search: electronic databases used to conduct literature searches included PubMed, Web of Science (February 15, 1983-September 29, 2019). Key words used to search titles and abstracts included renal cell carcinoma OR RCC OR advanced RCC OR metastatic RCC, AND radiotherapy OR palliative radiotherapy OR SBRT OR stereotactic radiosurgery. Retrieved studies included randomized controlled trials, retrospective and prospective studies, and meta-analyses.

\section{Palliative RT}

RCC has traditionally been considered to be radioresistant. Therefore, RT has played only a limited role in the treatment of RCC to date. A recent review concluded that neoadjuvant RT should not be recommended as a routine treatment for patients with locally advanced or unresectable RCC, because it did not improve the actuarial survival (10). According to the NCCN guidelines, adjuvant RT after nephrectomy also failed to show benefit for patients, even those with nodal involvement or partial resection (6).

However, conventional RT is a useful palliative therapeutic option for relieving the symptoms of patients with metastatic lesions. Results from several studies indicated that RT could relieve pain by bone metastasis in RCC patients. In 1983, Halperin et al. reported a clinical trial of RT for pain relief in RCC patients with 36 metastatic bone sites. The treatment response rate was $77 \%$ (28/36 sites); of the 28 sites that showed a response, 24 (86\%) were partially or completely free of pain for the remainder of the patients' lives (11). Similar results were reported in another retrospective study involving 34 patients with painful spinal metastases from RCC who were treated with conventional external beam RT (8-30 Gy in 1-10 fractions); the overall pain response rate was $68 \%(12)$. Wilson $e t$ al. conducted a retrospective study about biological effective dose (BED) for palliative RT treatment in mRCC. One hundred and forty-three independent metastatic sites were included, and the overall response rate was $73 \%$. The BED was based on the calculation of the $\alpha / \beta$ ratios of 3 and 7 Gy (BED 3 and BED 7, respectively). Comparison showed that relief of bone pain was more likely to be durable than the alleviation of symptoms at other metastatic sites. This article also concluded that it was not appropriate to use a higher BED dose as an indicator to predict response or sustained response in the palliative treatment of RCC (13).

A phase II study was conducted to evaluate the effect of RT on the symptoms of 31 patients with mRCC. Bone pain $(n=24)$ was the most common symptom reported, with the most commonly employed schedule being $30 \mathrm{~Gy} / 10$ fractions. After a mean follow-up time of 4.3 months, the authors reported that 19 (83\%) of 23 evaluable patients showed site-specific pain relief after treatment with RT (14). A later systematic review supported this result; with $30 \mathrm{~Gy}$ in 10 fractions as the most common radiation dose, $73.6 \%$ of patients with metastatic bone disease from RCC gained significant pain control (15).

\section{SBRT or SRS for advanced RCC}

Emerging evidence indicates that high-dose single-fraction RT can overcome the radioresistance of RCC and SBRT, which delivers high-dose single-fraction irradiation, has 
high rate of local control (LC) for both primary and metastatic RCC.

\section{Biological mechanism}

In a review article (16), De Meerleer et al. showed that CFRT induced tumor cell death mostly by oxygendependent DNA damage (double-strand breaks, or unrepaired or misrepaired breaks). But in about $60 \%$ of RCC cases, a tumor suppressor gene, the von Hippel Lindau (VHL), was found to lose function (16). Mutations in VHL had been found to result in the up-regulation of hypoxia-inducible genes (17). Under hypoxic conditions, HIF1 $\alpha$ couldn't go through ubiquitylation and proteasomal degradation and accumulated. This accumulation resulted in the upregulation of some related proangiogenic factors, such as vascular endothelial growth factor (VEGF) and fibroblast-derived growth factor, and downregulation of angiogenic inhibitors. These factors played an important role in protecting the endothelium of tumor cells, which was responsible for radioresistance (16). Under this circumstance, the effect of CFRT was limited.

This article also reviewed radiobiological difference between low and high dose per fraction of radiation and explained the biological principle underlying the phenomenon of RCC's sensitivity to SBRT. The authors suggested that SBRT overcame radioresistance mostly via the ASMase pathway (15). They reported that singlefraction SBRT (e.g., 15 Gy) results in the translocation of ASMase from intracellular compartments, such as lysosomes, to the outer leaflet of the plasma membrane, where it is captured in sphingolipid-enriched and cholesterol-enriched membrane micro-domains called rafts. During the hydrolysis of sphingomyelin by ASMase, ceramide, a pro-apoptotic messenger, is produced, which leads to endothelial apoptosis. Thus, ceramide plays a prime role in radiation-induced cell death in RCC. However, the specific mechanism of ASMase translocation to the outer leaflet of the plasma membrane requires further research.

In another experiment, Zhou et al. also hypothesized that hypoxia-inducible factor $2 \alpha$ (HIF $2 \alpha$ ) markedly activated the SHH-GLI1 signaling pathway, which was critical for embryonic development, leading to the radioresistance of RCC (18). The specific mechanism still needed to be further studied. Now, there are only three FAD-approved drugs for cancers that affect the Shh pathway. Two of compounds, vismodegib and sonidegib, target Smoothened (SMO). The other approved compound is arsenic trioxide
(ATO), which can suppress this pathway at the level of the GLI proteins, although current evidence suggests it also has other targets. But none of them apply to RCC (19).

\section{SBRT exerts prominent local therapeutic effect and has low toxicity for primary RCC patients}

Several prospective trials have attempted to evaluate the efficacy of SRS or SBRT for primary RCC. Pham et al. (20) carried out a phase I trial of SBRT for primary inoperable RCC. Eleven patients (gross tumor diameter $\geq 5 \mathrm{~cm}$ ) underwent treatment with 42 Gy/3 fractions and 9 patients (gross tumor diameter $<5 \mathrm{~cm}$ ) received a single fraction of 26 Gy. The author concluded for the first time that a threedimensional conformal radiological technique of $8-10$ beam could safely deliver highly stereotactic ablation to primary lesion targets. No grade 3 or 4 toxicities were reported during the 6-month follow-up period. Other prospective clinical trials have applied a similar regimen $(42 \mathrm{~Gy} / 3 \mathrm{f}$ for tumor size in diameter $\geq 5 \mathrm{~cm}, 26 \mathrm{~Gy} / 1 \mathrm{f}$ for tumor size $<5 \mathrm{~cm}$ ). The results demonstrated relationship between renal dysfunction and high-dose regions. Although SBRT results in a clinically acceptable decrease in renal function after 1 year (21). Furthermore, Siva et al. (22) showed that stereotactic ablative radiotherapy (SABR) was well tolerated by patients with small and large primary RCC. Within 2 years follow-up period, none of the patients had experienced local progression. The distant progression free and the overall survival (OS) rates were $89 \%$ and $92 \%$. No grade $4-5$ toxicities were recorded and $18 \%$ of patients reported no related toxicity. Next, two other doseescalation studies of SBRT for inoperable patients showed that dose level of $48 \mathrm{~Gy} / 4 \mathrm{f}$ and $35 \mathrm{~Gy} / 5 \mathrm{f}$ successfully achieved a satisfactory response, yielded acceptable toxicity, and preserved renal function $(23,24)$. A dose of 60 Gy in 3 fractions would be further evaluated in the next experimental research study. To observe the change in tumor size over time, Funayama et al.'s prospective study (25) enrolled 13 patients with primary RCC lesions, who were treated with SBRT. All of the renal tumors shrank slowly but continuously for years after SBRT. The 3-year local progression-free survival (PFS) rate was $92.3 \%$. The 2 and 3 -year OS rates were $91.7 \%$ and $71.3 \%$, respectively. However, the above clinical trials had several limitations. Firstly, the amount of enrolled patients was insufficient, and secondly, the median follow-up was generally limited.

In addition to the prospective trials, a meta-analysis study from IROCK (26) analyzed and compared the 
therapeutic effects of single-fraction (median dose, $25 \mathrm{~Gy}$ ) and multifraction (range 24-27 Gy, 2-10f) SABR. The two cohorts had similar ideal local cancer control. The 2-year and 4-year LC rate was both $97.8 \%$. However, singlefraction SABR could obtain better PFS and cancer-specific survival $(C S S)$ than a multifraction schedule $(\mathrm{P}<0.05)$. The authors also inferred that patients who received multifraction SABR were more inclined to progress in distant organs or to die due to cancer-related causes.

In 2019, another meta-analysis (27) reported that SABR was feasible for primary RCC, with a high LC rate. It analyzed 26 studies (including 11 prospective trials) involving 372 patients with 383 lesions, most of whom were diagnosed as inoperable. Radiation schedules of $26 \mathrm{~Gy} / 1 \mathrm{f}$ and $40 \mathrm{~Gy} / 5 \mathrm{f}$ were the most common. The LC rate was $97.2 \%$, and the rate of grade $3-4$ toxicities was $1.5 \%$. The authors suggested that SABR was an effective treatment for primary RCC and it was the preferred option for larger or centrally located tumors near the renal pelvic. Even for elder patients or patients with more comorbidities or patients with a higher risk of chronic kidney disease (CDK) progression, SABR was a reasonable treatment after an initial period of active surveillance (AS).

\section{SBRT can improve the LC rate for extracranial metastases, especially for oligometastasis}

Some reports suggested that complete metastasectomy could improve the CSS and quality of life of patients with locally metastatic lesions. However, local treatment is controversial $(28,29)$. In recent years, SBRT has emerged as a non-invasive alternative treatment for cases that are technically difficult to treat with surgery or for selected RCC patients with limited metastases $(28,30)$. Common sites of extracranial metastatic disease include the lungs and bones. Several clinical reports have shown SBRT to have a high LC rate and acceptable toxicity for treating metastases. Svedman et al. (30) reported a prospective phase II trial in which 82 lesions (including 63 in the lung/ mediastinum) were treated with stereotactic RT. The overall median follow-up time was 52 months. The results showed a high rate of LC (98\%) and low toxicity. For spinal metastases, RT had traditionally been used as a palliative therapy; However, more and more studies found that the effect of SBRT was far better than that of CFRT. Amini et al. (31) compared SBRT with conventional fractionated external beam RT (CF-EBRT) in patients with RCC spinal metastases. The control rates of SBRT and CF-EBRT at 10,
12 , and 24 months after treatment were $74.9 \%$ versus (vs.) $44.1 \%, 74.9 \%$ vs. $39.9 \%$, and $74.9 \%$ vs. $35.7 \%$, respectively. A significant difference was observed in the symptom control rates between the two treatments $(\mathrm{P}=0.020)$. A phase I/II study included 74 spinal metastatic lesions treated with SBRT, and the results indicated SBRT to be a safe and effective treatment for spinal metastases. Seventyfour spinal metastatic lesions were measured, of which the median tumor volume was $37.4 \mathrm{~cm}^{3}$ (range, $1.6-358 \mathrm{~cm}^{3}$ ). The 1-year PFS rate was $84 \%$ for all involved lesions (32). Another prospective study (33) proposed that different BEDs may account for improved LC. Single-fraction spinal SRS (24 Gy in 1f) was associated with improved LC compared with multifraction $(27 \mathrm{~Gy} / 3 \mathrm{f}$ or $30 \mathrm{~Gy} / 5 \mathrm{f})$ and should be considered in the upfront management of patients with RCC spinal metastases.

SBRT is also applied more in oligometastatic RCC. Oligometastasis is described as an intermediate condition in which the number of metastatic lesions is limited, involving either single or multiple organs (34). Some retrospective trials have demonstrated that patients with 1-3 metastases treated with SBRT had fewer distant recurrences and a longer survival time than patients with $>3$ metastatic sites $(35,36)$. Another study (37) reported that because SABR had a 1 -year LC rate of $91.2 \%$, as well as minimal acute and late complications, it should be considered as a treatment option for RCC patients with a limited number of metastatic sites.

\section{SRS has a bigh LC rate for brain metastasis, especially for smaller lesions}

The brain is one of the most common metastatic sites in RCC, and the survival of patients with BM is always poor (38). Advancements in imaging technology such as magnetic resonance imaging (MRI) and the longer surviving period of RCC patients have seen an increase in the incidence of BM (39). Nevertheless, there is no standard therapeutic regimen for BM. Treatment options include corticosteroids, surgery, whole-brain radiotherapy (WBRT), and SRS. Surgery is not frequently advocated for patients with multiple sites of intracranial disease or widespread metastatic disease. Marek et al. reported that patients who received WBRT alone had a high rate of local brain tumor progression and further emphasized the importance of radiosurgery. WBRT combined with radiosurgery was suitable for newly diagnosed and recurrent BM. It was also suggested that survival was not 
an accurate endpoint for evaluating treatment of BM. Symptom control can greatly improve the quality of life of patients with BM (40). Surgical removal of a solitary brain metastasis followed by WBRT has been found to be superior to WBRT alone, and WBRT alone should be reserved for patients with multiple metastases $(41,42)$. Furthermore, a systematic review concluded that there was no significant difference in OS comparing surgery plus WBRT vs. SRS plus WBRT in patients with single or solitary BM (43).

SRS is emerging as an attractive and effective treatment modality for newly diagnosed BM in patients with radioresistant malignant tumors, such as melanoma and RCC $(42,44,45)$. The 1-year LC rate for RCC BM treated with SRS is 91\% (45). Studies have found that smaller tumor volume is a positive predictor of response in RCC $(38,44)$. Consistent with this, a systematic review revealed that LC was significantly correlated with the volume of metastases. The LC rates for BM at 1 and 2 years after treatment were $91.8 \%$ and $86.1 \%$, respectively; however, the LC rate declined for larger tumors $(>2 \mathrm{~cm})$. Survival was not found to be statistically different between patients with a different number of metastases $(<5$ versus $\geq 5 \mathrm{BM}$ ) (46). Whether or not WBRT should be combined with SRS in the treatment of radioresistant BM remains controversial (43). A phase II trial conducted by Rafael et al. showed the 3- and 6-month intracranial failure rates without WBRT to be $25.8 \%$ and $48.3 \%$, respectively. However, the generalizability of these results is limited due to the small number of patients involved $(n=31)(42)$. A retrospective clinical trial showed that the median OS times between SRS and SRW + WBRT (12 vs. 16 months, respectively) had no statistical difference, but the addition of WBRT to SRS increased the intracerebral control rate in patients with one to three cerebral lesions (47). A similar result was reported in another retrospective study, in which patients with 1-3 BM were involved. The authors suggested that additional WBRT was justified, particularly in recursive partitioning analysis (RPA) class I patients (48).

The recent studies for BM mostly involved non-RCC patients. Further prospective research involving RCC patients with BM is urgently needed.

\section{SBRT combined with systemic therapies}

NCCN Guidelines recommend that systemic therapies are the current standard treatment strategies in clear cell histology with poor-risk features for advanced RCC or
mRCC (6). However, we found some clinical evidence that SBRT combined with systemic therapy has a synergistic antitumor effect on RCC.

\section{Concurrent SBRT and targeted therapy is tolerable and has a synergistic antitumor effect on RCC}

Tyrosine kinase inhibitors (TKIs) and anti-VEGF antibodies, which are the most commonly used targeted therapy, are now widely used as first- and second-line treatments for advanced RCC. Anticancer drugs targeting the mammalian target of rapamycin (mTOR) are also widely used. The United States Food and Drug Administration (FDA) has approved a number of targeted agents as first or subsequent lines of treatment for advanced RCC including sunitinib, sorafenib, pazopanib, axitinib, temsirolimus, and everolimus (6).

Endothelial angiogenesis and a paucity of endothelial apoptotic response are considered to be the likely main causes of radiation resistance. Endothelial apoptosis is the key to radiation-induced cell death in RCC (16). Therefore, antiangiogenic drugs, such as TKIs, are theoretically considered to be radiosensitizers. Currently, the clinical evidence of the effect of concurrent targeted drug therapy and RT on RCC is limited and inconsistent. Most studies to date have used sunitinib, which is a small molecule receptor TKI that targets multiple pathways including receptor tyrosine kinase (KIT), vascular endothelial growth factor receptor (VEGFR1, VEGFR2, VEGFR3), platelet derived growth factor receptor a (PDGFRa), Fms-like tyrosine kinase-3 receptor (FLT3), and the receptor encoded by the ret proto-oncogene (RET) (49).

A phase 1 dose-escalation study of concurrent sunitinib and image-guided radiotherapy (IGRT) focused on the safety and maximum tolerated dose. The authors proposed that the addition of sunitinib $(25-37.5 \mathrm{mg})$ to IGRT was tolerable in selected subjects with 1-5 metastatic sites and did not potentiate RT toxicity (49). A phase II trial focusing on concurrent sunitinib and IGRT for oligometastases was next carried out. The treatment schedule was $37.5 \mathrm{mg}$ sunitinib daily (days $1-28$ ) and IGRT 50 Gy (days 8-12 and 15-19). The 18-month LC, distant control, PFS, and OS rates were $75 \%, 52 \%, 56 \%$, and $71 \%$, respectively (50). Similarly, more specific data in another study of the same treatment showed that the 4-year LC, distant control, PFS, and OS rates were 75\%, $40 \%, 34 \%$, and $29 \%$, respectively (51). Another study involved 41 patients with cerebral or spinal metastatic 
lesions from RCC who were treated synchronously with sunitnib and hypo-fractionated high-dose RT. Concurrent SRS and anti-angiogenic therapy was confirmed to be a non-invasive, safe, and efficient treatment for patients with spinal or cerebral metastasis from RCC (52). Kusuda et al. reviewed the effect of the combination sunitinib with RT in five RCC patients with BM. Sunitinib was administered after RT, and all patients displayed shrinkage of the BM. Treatment-related adverse events did not differ significantly between patients treated with the combination of sunitinib with RT and those who received sunitinib alone. Moreover, four out of the five patients with BM had a long survival period, with OS of 10.5 , $12.5,20.8$, and 21.5 months, respectively. The result of the study demonstrated that concurrent sunitinib and SRS could be safely employed to treat RCC patients with BM and yielded favorable prognostic outcomes. However, four of the five patients showed disease progression of BM. Therefore, to acquire more convincing results, more cases are needed (53). Another retrospective study reported sequential therapy combined with targeted therapies followed by RT for mRCC. Only seven cases were enrolled and the response of metastatic lesions to the treatment was obvious (54). A larger-scale retrospective study involving $362 \mathrm{RCC} \mathrm{BMs}$, of which $65 \%$ were treated with SRS combined with TKIs, reported 12- and $36-$ month OS rates to be $52 \%$ and $29 \%$, respectively. The median OS was 13.5 months (95\% CI, 11-20 months). This result suggested that concurrent TKI and SBS treatment could be a promising approach to optimize LC of mRCC, without increasing neurologic toxicity (55).

Pazopanib is also a first-line TKI, targeting VEGFR. De Wolf et al. reported a phase I dose-escalation experiment combining with high-dose RT and pazopanib in mRCC. The radiation dose was escalated in three dose levels (24 Gy/3, $30 \mathrm{~Gy} / 3$, and $36 \mathrm{~Gy} / 3$ ). The results showed that the maximum tolerated dose was not reached, which provided evidence that the combination treatment had a synergistic antitumor effect. The patients, who had inoperable localized disease or limited metastatic disease, initially achieved an ideal curative effect. The 1-year LC and PFS rates were $83 \%$ and $28 \%$, respectively (56).

Everolimus is another type of targeting drug for mRCC, which is an orally available analogue of rapamycin that inhibits the activation of the mTOR pathway. A case report described that a patient with mRCC gained radiological complete response (CR) after treatment with radiation (total dose: 30 Gy in 10 fractions) and everolimus (10 mg/daily).
The treatment was well tolerated and the patient presented only limited mucositis, and moderate hypertriglyceridemia and hypercholesterolemia (57).

A recent retrospective study by $\mathrm{He}$ et al. reported outcomes of 350 consecutive patients with mRCC who were treated with SBRT combined with TKIs. The study focused on survival benefits and the timing of SBRT combined with targeted treatment. The median OS was 61.2 months, and the median PFS was 11.5 months. The 2 -year LC rate reached 94\%. CR was obtained in 16 (34\%) lesions in patients irradiated before TKI failure, but in only $4(7 \%)$ lesions in patients who were irradiated after TKI failure $(\mathrm{P}=0.001)$. Four (7\%) patients experienced grade 3 toxicities. Therefore, in the treatment with TKIs, SBRT could achieved better PFS, OS, and CR rates. It should be delivered before the failure of TKI therapy, but not after (58).

\section{Immunotherapy and SBRT bave a synergistic antitumor effect}

The standard of treatment for mRCC is systemic therapy. In recent years, immune checkpoint inhibitors (ICIs), including anti-cytotoxic $\mathrm{T}$ lymphocyte antigen-4 (antiCTLA-4) and anti-programmed cell death-1 (anti-PD-1)/ PD-L1 blockers, have emerged as a popular treatment for several advanced or metastatic solid tumors.

Programmed cell death-1 (PD-1) checkpoint inhibitors selectively block the PD-1/PD-1 ligands 1 and 2 pathway. In November 2015, nivolumab was the first ICI approved by the FDA as a therapy for patients with advanced RCC (59).

In the randomized, open-label, phase III CheckMate 025 study (NCT01668784), nivolumab was demonstrated to shrink tumors and to effectively improve symptoms for advanced RCC patients (60). However, the number of patients who achieved remarkable outcomes was limited. Still, a considerable proportion of patients did not obtain a clinically effective response. Also, some patients who initially obtained a durable response to ICI therapy showed significant progression in the follow-up , and more than $55 \%$ of RCC patients who did not receive combined treatments developed resistance to different immunotherapies $(59,61)$. These findings were supported by the results of Chowdhry et al.'s study. Nivolumab alone had limited efficacy in the treatment of BM. But Nivolumab with either RT or surgery had been strongly suggested to achieve more satisfactory efficacy and LC (62). 
Some scholars have put forward the hypothesis that RT and immunotherapy have a synergistic antitumor effect, with preclinical evidence showing that immunotherapy combined with RT increased loco-regional and systemic disease control (63-65).

The combination of RT and immunotherapy is based on the abscopal effect, which results from immune activation by RT and accounts for the regression of metastatic cancer at distant sites with no exposure to radiation (66). The CR and abscopal effects of RCC treated with ICI and RT have mostly been described in case reports (67). Multiple clinical trials of immunotherapy combined with RT in the treatment of RCC are ongoing.

\section{Conclusions}

Conventional fractionated RT has traditionally been used as a palliative treatment to relieve pain and has a limited effect on LC. SBRT has a high LC rate for advanced or mRCC and could be used as an alternative to surgery for oligometastatic RCC patients. It is safe and tolerable to combine SBRT with systemic therapies. Concurrent immunotherapy and SBRT is a promising therapeutic strategy for patients with advanced or mRCC. However, evidence from clinical trials is still limited, and the efficacy, proper timing, and safety of the combination of immunotherapy and RT need to be further explored.

\section{Acknowledgments}

Funding: Medical Science Research Project of Dalian (Project No. 1912035).

\section{Footnote}

Reporting Checklist: The authors have completed the Narrative Review reporting checklist. Available at http:// dx.doi.org/10.21037/tau-20-1466

Conflicts of Interest: All authors have completed the ICMJE uniform disclosure form (available at http://dx.doi. org/10.21037/tau-20-1466). The authors have no conflicts of interest to declare.

Ethical Statement: The authors are accountable for all aspects of the work in ensuring that questions related to the accuracy or integrity of any part of the work are appropriately investigated and resolved.
Open Access Statement: This is an Open Access article distributed in accordance with the Creative Commons Attribution-NonCommercial-NoDerivs 4.0 International License (CC BY-NC-ND 4.0), which permits the noncommercial replication and distribution of the article with the strict proviso that no changes or edits are made and the original work is properly cited (including links to both the formal publication through the relevant DOI and the license). See: https://creativecommons.org/licenses/by-nc-nd/4.0/.

\section{References}

1. Ng CS, Wood CG, Silverman PM, et al. Renal cell carcinoma: diagnosis, staging, and surveillance. AJR Am J Roentgenol 2008;191:1220-32.

2. Kunkle DA, Haas NB, Uzzo RG. Adjuvant therapy for high-risk renal cell carcinoma patients. Curr Urol Rep 2007;8:19-30.

3. Wang L, Cai W, Kong W, et al. Plasma fibrinogen as prognostic predictor in patients with metastatic renal cell carcinoma receiving target therapy. Transl Cancer Res 2018;7:1384-92.

4. Yang DC, Chen CH. Potential New Therapeutic Approaches for Renal Cell Carcinoma. Semin Nephrol 2020;40:86-97.

5. Mollica V, Di Nunno V, Massari F. Pembrolizumab plus axitinib: a new treatment option for patients with metastatic renal cell carcinoma. Chin Clin Oncol 2019;8:S21.

6. Motzer RJ, Jonasch E, Michaelson MD, et al. NCCN Guidelines Insights: Kidney Cancer, Version 2.2020. J Natl Compr Canc Netw 2019;17:1278-85.

7. Lo SS, Fakiris AJ, Chang EL, et al. Stereotactic body radiation therapy: a novel treatment modality. Nat Rev Clin Oncol 2010;7:44-54.

8. Ning S, Trisler K, Wessels BW, et al. Radiobiologic studies of radioimmunotherapy and external beam radiotherapy in vitro and in vivo in human renal cell carcinoma xenografts. Cancer 1997;80:2519-28.

9. Demaria S, Golden EB, Formenti SC. Role of Local Radiation Therapy in Cancer Immunotherapy. JAMA Oncol 2015;1:1325-32.

10. Fusco V, Parisi S, d'Andrea B, et al. Role of radiotherapy in the treatment of renal cell cancer: updated and critical review. Tumori 2017;103:504-10.

11. Halperin EC, Harisiadis L. The role of radiation therapy in the management of metastatic renal cell carcinoma. Cancer 1983;51:614-7. 
12. Hunter GK, Balagamwala EH, Koyfman SA, et al. The efficacy of external beam radiotherapy and stereotactic body radiotherapy for painful spinal metastases from renal cell carcinoma. Pract Radiat Oncol 2012;2:e95-100.

13. Wilson D, Hiller L, Gray L, et al. The effect of biological effective dose on time to symptom progression in metastatic renal cell carcinoma. Clin Oncol (R Coll Radiol) 2003;15:400-7.

14. Lee J, Hodgson D, Chow E, et al. A phase II trial of palliative radiotherapy for metastatic renal cell carcinoma. Cancer 2005;104:1894-900.

15. Ganju RG, TenNapel M, Mahan N, et al. The Efficacy of Conventionally Fractionated Radiation in the Management of Osseous Metastases from Metastatic Renal Cell Carcinoma. J Oncol 2018;2018:6384253.

16. De Meerleer G, Khoo V, Escudier B, et al. Radiotherapy for renal-cell carcinoma. Lancet Oncol 2014;15:e170-7.

17. Cockman ME, Masson N, Mole DR, et al. Hypoxia inducible factor-alpha binding and ubiquitylation by the von Hippel-Lindau tumor suppressor protein. J Biol Chem 2000;275:25733-41.

18. Zhou J, Wu K, Gao D, et al. Reciprocal regulation of hypoxia-inducible factor $2 \alpha$ and GLI1 expression associated with the radioresistance of renal cell carcinoma. Int J Radiat Oncol Biol Phys 2014;90:942-51.

19. Carpenter RL, Ray H. Safety and Tolerability of Sonic Hedgehog Pathway Inhibitors in Cancer. Drug Saf 2019;42:263-79.

20. Pham D, Thompson A, Kron T, et al. Stereotactic ablative body radiation therapy for primary kidney cancer: a 3-dimensional conformal technique associated with low rates of early toxicity. Int J Radiat Oncol Biol Phys 2014;90:1061-8.

21. Siva S, Jackson P, Kron T, et al. Impact of stereotactic radiotherapy on kidney function in primary renal cell carcinoma: Establishing a dose-response relationship. Radiother Oncol 2016;118:540-6.

22. Siva S, Pham D, Kron T, et al. Stereotactic ablative body radiotherapy for inoperable primary kidney cancer: a prospective clinical trial. BJU Int 2017;120:623-30.

23. Ponsky L, Lo SS, Zhang Y, et al. Phase I dose-escalation study of stereotactic body radiotherapy (SBRT) for poor surgical candidates with localized renal cell carcinoma. Radiother Oncol 2015;117:183-7.

24. Correa RJM, Ahmad B, Warner A, et al. A prospective phase I dose-escalation trial of stereotactic ablative radiotherapy (SABR) as an alternative to cytoreductive nephrectomy for inoperable patients with metastatic renal cell carcinoma. Radiat Oncol 2018;13:47.

25. Funayama S, Onishi H, Kuriyama K, et al. Renal Cancer is Not Radioresistant: Slowly but Continuing Shrinkage of the Tumor After Stereotactic Body Radiation Therapy. Technol Cancer Res Treat 2019;18:1533033818822329.

26. Siva S, Louie AV, Warner A, et al. Pooled analysis of stereotactic ablative radiotherapy for primary renal cell carcinoma: A report from the International Radiosurgery Oncology Consortium for Kidney (IROCK). Cancer 2018;124:934-42.

27. Correa RJM, Louie AV, Zaorsky NG, et al. The Emerging Role of Stereotactic Ablative Radiotherapy for Primary Renal Cell Carcinoma: A Systematic Review and MetaAnalysis. Eur Urol Focus 2019;5:958-69.

28. Wang CJ, Christie A, Lin MH, et al. Safety and Efficacy of Stereotactic Ablative Radiation Therapy for Renal Cell Carcinoma Extracranial Metastases. Int J Radiat Oncol Biol Phys 2017;98:91-100.

29. Dabestani S, Marconi L, Hofmann F, et al. Local treatments for metastases of renal cell carcinoma: a systematic review. Lancet Oncol 2014;15:e549-61.

30. Svedman C, Sandström P, Pisa P, et al. A prospective Phase II trial of using extracranial stereotactic radiotherapy in primary and metastatic renal cell carcinoma. Acta Oncol 2006;45:870-5.

31. Amini A, Altoos B, Bourlon MT, et al. Local control rates of metastatic renal cell carcinoma (RCC) to the bone using stereotactic body radiation therapy: Is RCC truly radioresistant? Pract Radiat Oncol 2015;5:e589-96.

32. Chang EL, Shiu AS, Mendel E, et al. Phase I/II study of stereotactic body radiotherapy for spinal metastasis and its pattern of failure. J Neurosurg Spine 2007;7:151-60.

33. Ghia AJ, Chang EL, Bishop AJ, et al. Single-fraction versus multifraction spinal stereotactic radiosurgery for spinal metastases from renal cell carcinoma: secondary analysis of Phase I/II trials. J Neurosurg Spine 2016;24:829-36.

34. Loh J, Davis ID, Martin JM, et al. Extracranial oligometastatic renal cell carcinoma: current management and future directions. Future Oncol 2014;10:761-74.

35. Wersäll PJ, Blomgren H, Lax I, et al. Extracranial stereotactic radiotherapy for primary and metastatic renal cell carcinoma. Radiother Oncol 2005;77:88-95.

36. Buti S, Bersanelli M, Viansone A, et al. Treatment Outcome of metastatic lesions from renal cell carcinoma underGoing Extra-cranial stereotactic body radiotherapy: The together retrospective study. Cancer Treat Res Commun 2020;22:100161.

37. Wang CJ, Christie A, Lin MH, et al. Safety and Efficacy 
of Stereotactic Ablative Radiation Therapy for Renal Cell

Carcinoma Extracranial Metastases. Int J Radiat Oncol Biol Phys 2017;98:91-100.

38. Wrónski M, Maor MH, Davis BJ, et al. External radiation of brain metastases from renal carcinoma: a retrospective study of 119 patients from the M. D. Anderson Cancer Center. Int J Radiat Oncol Biol Phys 1997;37:753-9.

39. Patchell RA. The management of brain metastases. Cancer Treat Rev 2003;29:533-40.

40. Fuller BG, Kaplan ID, Adler J, et al. Stereotaxic radiosurgery for brain metastases: the importance of adjuvant whole brain irradiation. Int J Radiat Oncol Biol Phys 1992;23:413-8.

41. Patchell RA, Tibbs PA, Walsh JW, et al. A randomized trial of surgery in the treatment of single metastases to the brain. N Engl J Med 1990;322:494-500.

42. Manon R, O'Neill A, Knisely J, et al. Phase II trial of radiosurgery for one to three newly diagnosed brain metastases from renal cell carcinoma, melanoma, and sarcoma: an Eastern Cooperative Oncology Group study (E 6397). J Clin Oncol 2005;23:8870-6.

43. Fuentes R, Osorio D, Expósito Hernandez J, et al. Surgery versus stereotactic radiotherapy for people with single or solitary brain metastasis. Cochrane Database Syst Rev 2018;8:CD012086.

44. Hanson PW, Elaimy AL, Lamoreaux WT, et al. A concise review of the efficacy of stereotactic radiosurgery in the management of melanoma and renal cell carcinoma brain metastases. World J Surg Oncol 2012;10:176.

45. Lwu S, Goetz P, Monsalves E, et al. Stereotactic radiosurgery for the treatment of melanoma and renal cell carcinoma brain metastases. Oncol Rep 2013;29:407-12.

46. Wardak Z, Christie A, Bowman A, et al. Stereotactic Radiosurgery for Multiple Brain Metastases From Renal-Cell Carcinoma. Clin Genitourin Cancer 2019;17:e273-80.

47. Fokas E, Henzel M, Hamm K, et al. Radiotherapy for brain metastases from renal cell cancer: should whole-brain radiotherapy be added to stereotactic radiosurgery?: analysis of 88 patients. Strahlenther Onkol 2010;186:210-7.

48. Rades D, Kueter JD, Hornung D, et al. Comparison of stereotactic radiosurgery (SRS) alone and whole brain radiotherapy (WBRT) plus a stereotactic boost (WBRT+SRS) for one to three brain metastases. Strahlenther Onkol 2008;184:655-62.

49. Kao J, Packer S, Vu HL, et al. Phase 1 study of concurrent sunitinib and image-guided radiotherapy followed by maintenance sunitinib for patients with oligometastases: acute toxicity and preliminary response. Cancer 2009;115:3571-80.

50. Tong CC, Ko EC, Sung MW, et al. Phase II trial of concurrent sunitinib and image-guided radiotherapy for oligometastases. PLoS One 2012;7:e36979.

51. Kao J, Chen CT, Tong CC, et al. Concurrent sunitinib and stereotactic body radiotherapy for patients with oligometastases: final report of a prospective clinical trial. Target Oncol 2014;9:145-53.

52. Staehler M, Haseke N, Nuhn P, et al. Simultaneous antiangiogenic therapy and single-fraction radiosurgery in clinically relevant metastases from renal cell carcinoma. BJU Int 2011;108:673-8.

53. Kusuda Y, Miyake H, Terakawa T, et al. Treatment of brain metastases from renal cell carcinoma with sunitinib and radiotherapy: our experience and review of the literature. Int J Urol 2011;18:326-9.

54. Gravis G, Faure M, Rybikowski S, et al. Radiation therapy following targeted therapy in oligometastatic renal cell carcinoma. Mol Clin Oncol 2015;3:1248-50.

55. Klausner G, Troussier I, Biau J, et al. Stereotactic Radiation Therapy for Renal Cell Carcinoma Brain Metastases in the Tyrosine Kinase Inhibitors Era: Outcomes of 120 Patients. Clin Genitourin Cancer 2019;17:191-200.

56. De Wolf K, Rottey S, Vermaelen K, et al. Combined high dose radiation and pazopanib in metastatic renal cell carcinoma: a phase I dose escalation trial. Radiat Oncol 2017;12:157.

57. Detti B, Francolini G, Becherini C, et al. Complete response in metastatic renal cell carcinoma after radiotherapy and everolimus: a clinical case and review of the literature. J Chemother 2016;28:432-4.

58. He L, Liu Y, Han H, et al. Survival Outcomes After Adding Stereotactic Body Radiotherapy to Metastatic Renal Cell Carcinoma Patients Treated With Tyrosine Kinase Inhibitors. Am J Clin Oncol 2020;43:58-63.

59. Ko EC, Formenti SC. Radiotherapy and checkpoint inhibitors: a winning new combination? Ther Adv Med Oncol 2018;10:1758835918768240.

60. Escudier B, Motzer RJ, Sharma P, et al. Treatment Beyond Progression in Patients with Advanced Renal Cell Carcinoma Treated with Nivolumab in CheckMate 025. Eur Urol 2017;72:368-76.

61. Sun X, Gan L, Na A, et al. Combination with Stereotactic Body Radiotherapy Offers a Promising Strategy to Overcome Resistance to Immunotherapy in Advanced 
Renal Cell Cancer. J Oncol 2019;2019:1483406.

62. Chowdhry AK, Hardy SJ, Milano MT. Nivolumab without brain radiotherapy is insufficient for the treatment of most patients with brain metastases from clear cell renal cell carcinoma. Ann Transl Med 2019;7:S366.

63. Deng L, Liang H, Burnette B, et al. Irradiation and antiPD-L1 treatment synergistically promote antitumor immunity in mice. J Clin Invest 2014;124:687-95.

64. Dovedi SJ, Adlard AL, Lipowska-Bhalla G, et al. Acquired resistance to fractionated radiotherapy can be overcome by concurrent PD-L1 blockade. Cancer Res 2014;74:5458-68.

65. Buttigliero C, Allis S, Tucci M, et al. Role of radiotherapy in improving activity of immune-modulating drugs in advanced renal cancer: Biological rationale and clinical evidences. Cancer Treat Rev 2018;69:215-23.

66. Lauko A, Thapa B, Venur VA, et al. Management of Brain Metastases in the New Era of Checkpoint Inhibition. Curr Neurol Neurosci Rep 2018;18:70.

67. Xie G, Gu D, Zhang L, et al. A rapid and systemic complete response to stereotactic body radiation therapy and pembrolizumab in a patient with metastatic renal cell carcinoma. Cancer Biol Ther 2017;18:547-51.

(English Language Editor: J. Reynolds)
Cite this article as: Wei Q, He H, Lv L, Xu X, Sun W. The promising role of radiotherapy in the treatment of advanced or metastatic renal cell carcinoma: a narrative review. Transl Androl Urol 2020;9(6):2821-2830. doi: 10.21037/tau-20-1466 